\title{
Performance and Analysis of Video Streaming of Signals in Wireless Network Transmission
}

\author{
Chinnusamy. $\mathrm{K}^{1}$ and Abinaya Preethi. $\mathrm{K}^{2}$ \\ ${ }^{l}$ Faculty of Engineering Department of Electronics and Communication Engineering Affiliated to Anna \\ University, Chennai Tamil Nadu, INDIA \\ ${ }^{2} P G$ Scholar Department of Electronics and Communication Engineering Affiliated to Anna University,
} Chennai Tamil Nadu, INDIA

\begin{abstract}
In this work, we describe an effective video communication for wireless transmission of H.264/AVC medical ultrasound video over mobile WiMAX networks. Medical ultrasound is encoded using error resilient encoding in which, the quantization levels are varied according to the function of the diagnostic significance of image region. We explain how our proposed system allows for the transmission of high-resolution clinical video which is being encoded at the clinical acquisition resolution and then decoded with low delay.

We perform this functional concept by using OPNET simulations of the mobile WiMAX medium and also considering some of the physical layer characteristics also such as service prioritization classes, different types of modulation being performed, fading channel's conditions, coding schemes and their types and mobility.We are encoding the ultrasound videos at $4 C I F(704 \times 576)$ resolution to provide high level of clarity. The video quality assessment is being done based on both of their evaluations in objective and subjective manner.
\end{abstract}

Index Terms: WiMAX, Long Term Evolution(LTE), High Efficiency Video Coding(HEVC), Flexible Macroblock Ordering (FMO), Diagnostically Relevant Encoding, 4G, mobile health care.

\section{Introduction}

Over the past decade, demand for mobile health systems has been growing[1]-[3].This demand is highly driven by the need for providing response in emergency conditions for remote diagnosis and care, giving proper medical education

Advancements in medical video coding, with emerging technologies are needed to support m-health video communication for clinical practice.The mobile health systems are expected to bring greater benefits to the elderly, people residing in remote areas, and also it improves the quality of life of patients with mobility problems.Researches in mobile health video communication system includes modality-aware diagnostically driven systems, which adapts to the wireless transmission medium.

Diagnostically driven systems rely on the use of diagnostic Regions Of Interest (ROI)[4]-[6] clinical video quality assessment methods are used for communicating the medical video to the medical expert[5],[8]. In terms of the wireless infrastructure, the mobile health video systems are based on 3G wireless networks[3],[5] which have a limited upload data rates of channels that are supported (upto 384kbps) associated with encoding parameters bounded to the CIF resolution video size.

For atherosclerotic plaque ultrasound video, shifting from QCIF $(176 \times 144)$ to CIF $(352 \times 288)$ resolution, it enables assessment of plaque type[6] and providing this clinical information to the medical expert for analyzing the possibility of a plaque rupture, leading to stroke. The previous studies did not mention about the issues associated with individual network parameters of high-resolution video transmission.

There is a great demand in $3.5 \mathrm{G}$ and $4 \mathrm{G}$ wireless technology[1] usage in facilitating medical video communication. The goal is to deliver high resolutions and video frames with low-delay and low packet loss rates (PLR) that provides an experience for in- hospital examination purpose.

In this work, we provide an added advantage of high resolution (4CIF-704×576) medical video communication over mobile Worldwide Interoperability for Microwave Access (WiMAX) networks for emergency telemedicine. The proposed end-to-end ultrasound video communication is based on clinical criteria. The clinical approach extends from CIF resolution to higher resolution of 4CIF and lower resolution of QCIF. In diagnostically relevant selection of encoding, the parameters based on video region's clinical importance was used with different medium access control (MAC) and some of the physical layer features of mobile WiMAX that supports the mobile health systems during emergency telemedicine.

The main goal of the network study is to provide a parameter selection of networks that accommodates different emergency situations under varying network state. Comparison of this proposed work in three different areas are as follows 


\section{A.Robust Video Encoding at three different resolutions}

We consider QCIF,CIF,\&4CIF resolutions and then discuss the spatial resolution associated with this criteria. Measures the improvement in terms of bitrate reduction, which is being used for increasing the peak signal-to-noise ratio(PSNR)of the reconstructed video as compared to H.264/AVC encoding. We use BD-PSNR algorithm, which provides average gain in bitrate equivalent of PSNR levels for 240 cases of CIF, QCIF and $4 \mathrm{CIF}$ resolutions. Beyond these cases, additional 1500 4CIF transmission cases over mobile WiMAX have been achieved.

\section{B.Relationship between spatial resolution and clinical diagnosis}

The clinical criteria has been addressed with different resolutions. For applicational usage, the additional resolution interms of higher order allows us to not only visualize the plaque type[7], it can also view the morphology of atherosclerotic plaque.

\section{C.Medical video communications over mobile WiMAX networks}

We propose a framework for mobile WiMAX video communication based on OPNET simulator. For this purpose, real ultrasound videos are used to generate the video files, which are imported to OPNET to model the wireless video transmission.

After the transmission, the video packets are mapped back to the original files for decoding[2]. This method allows us to configure both objectively and subjectively. Based on the previous method, we provide the recommendations for mobile WiMAX network parameter utilization for improving the capacity of the communicated video

Mobile WiMAX network's performance can be analyzed using the quality of service measurements such as PLR, packet delay and PSNR of reconstructed video bitstreams. The emergency telemedicine situations are handled by the three different channel modulation and coding schemes, hybrid signal attenuation model and distances from the base station(BS)and various mobility patterns.

\section{Mobile Wimax For Video Communication}

WiMAX medium for fixed wireless applications was the first to be standardized by IEEE 802.16-in the year 2004 and then it has been expanded to be used in other applications like mobile in 2005 by the IEEE 802.16e standard. Now, recent standard of $802.16 \mathrm{~m}$ which is also known as wireless MAN-Advanced is considering to be in usage of the $4 \mathrm{G}$ technology[3].

\section{A.Physical Layer Features}

The primary features of the physical layer involves the coding of various techniques(QPSK,16QAM,64-QAM)adaptive modulation schemes, hybrid automatic repeat request(hARQ) and fast channel feedback. WiMAX uses OFDM access that primarily divides the transmission bandwidth into multiple subcarriers where their number ranges from 128 for $1.25 \mathrm{MHz}$ channel bandwidth and the range extends upto 2048 for $20 \mathrm{MHz}$ channels. The orthogonality phenomenon among these subcarriers leads to overlapping resulting finally in fast fading.

The multipath interference is being employed by OFDM, which separates the available bandwidth and allocates it to all the requested parallel applications for the improvement of the system efficiency. MIMO antenna system improves the communication performance that provides increases in throughput of data and link range, without any additional bandwidth requirements (or) increase in transmission of power.

\section{B.MAC Layer Features}

MAC layer provides many features that is of QoS[5],[6] with different prioritization classes, scheduling upper link and downward link with efficient mobility management and security.

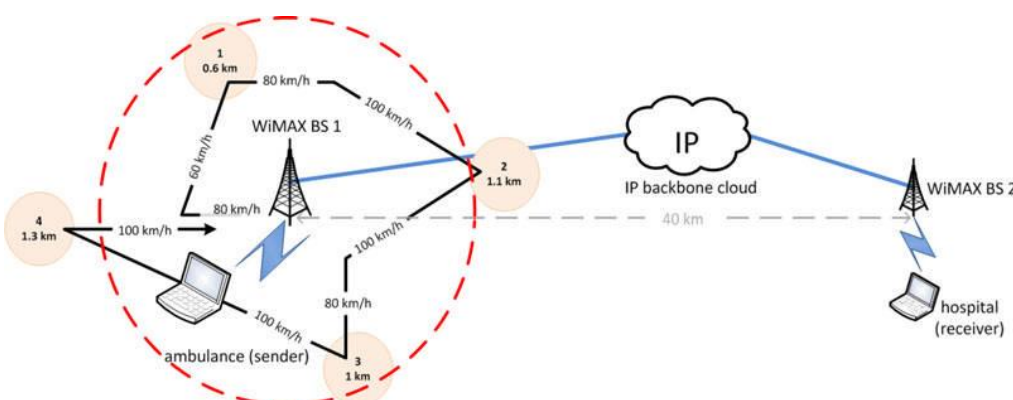

Fig.1.The ambulance travels with speeds ranging from 60 to $100 \mathrm{~km} / \mathrm{h}$, topology for the medical video transmission over mobile WiMAX networks 
For real time video streaming, best is to use the real-time polling service(rtps) QoS class. This rtps class provides specifications for minimum data rate, maximum latency and traffic priority. Mobility management is being well developed in $802.16 \mathrm{e}$ and in $802.16 \mathrm{~m}$ standards[4], which was an issue in $802.16 \mathrm{~d}$ for fixed connections.

\section{Methodology}

The methodology provides that the usage of high-resolution medical video communication performance over mobile WiMAX networks based on realistic clinical scenarios. One main aim is to develop a real video scenarios that is used to develop mobile(m-health)health video systems for the emergency purposes. The main concept is to communicate the patient's video to the hospital for diagnosis and assistance with inambulance care. Who can complete their hospital's admission related tasks(surgery preparations).

The scenario depicted in the work faces more challenges in wireless communication of ultrasound video of reliable quality where this depicts the route from emergency area to the hospital by which the patient has been given the paramedics with those equipments present inside the ambulance.

\section{A.Preprocessing}

To match the bandwidth that is being available, the resolution of the video and frame-rates has to be adjusted. The mobile WiMAX networks with high bandwidth, allows the transmission of 4CIF video resolution at the rate of 15 frames/s.

\section{1)Diagnostically Relevant Encoding}

This system uses diagnostically driven relevant and resilient encoding scheme. Each of the video is assigned with a quality level based on its diagnostic significance. The quality levels are being implemented by adjusting the values of the quantization parameter. The (nondiagnostically important region)background is compressed to reduce the bitrate requirements[3],[4],[7].

\section{2)Plaque region for visualizing plaque type, morphology and motion}

This is the primary form of Region Of Interest. The stability of the plaque can be accessed only by visualizing the type and morphology of the for critical areas(see Table II).

\section{3)Near and far wall regions for visualizing the wall motion}

The interest in visualizing the near and far walls comes from the need to compare these motion patterns of the plaque. Different patterns will be observed for unstable plaques.

\section{4)ECG region for visualizing ECG waveform}

ECG waveform is mainly used to visualize the plaque and stenosis that predicts changes in different parts of the cardiac cycle. We are considering three different video resolutions of formats mainly QCIF (176 $\times 144)$, CIF $(352 \times 288)$ and 4CIF $(704 \times 576)$. The main point of view is to provide a scalable high resolution over WiMAX networks which is mainly of 4CIF formats. To evaluate the proposed concept, a series of ten videos are being encoded at 15 frames/s. For the purpose of encoding JM H.264/AVC reference software has been used.

\section{B.Mobile WiMAX Video Transmission}

This mainly imparts in the transmission of ultrasound video and identifying the possible degradation while transmitting from the ambulance to the hospital(see Fig.1). For this purpose, we refer the usage of coding schemes, different channel modulation, distance from the base station and the availability of bandwidth.

\section{C.Video Quality Assessment}

This includes both objective and subjective evaluations. Clinical evaluation is being performed by the relevant medical expert (see Table II) whereas objective video evaluation techniques are mathematical models and are automatically evaluated by a computer program. In this PSNR is the most widely used objective video quality metrics.

TABLE I

Average bitrate gain of diagnostically relevant encoding

\begin{tabular}{cccc}
\hline \hline & QCIF & CIF & 4CIF \\
\hline Plaque Detection & $\sqrt{ }$ & $\sqrt{ }$ & $\sqrt{ }$ \\
Artery Stenosis & $\sqrt{ }$ & $\sqrt{ }$ & $\sqrt{ }$ \\
Plaque Type & & $\sqrt{ }$ & $\sqrt{ }$ \\
Plaque Morphology & & & $\sqrt{ }$ \\
\hline \hline
\end{tabular}




\section{Hardware And Software Tools}

Ultrasound video can be acquired by a portable device. Encoding is being done by using the JM H.264/AVC reference software.JM is the current best-established software for H.264/AVC encoding and decoding. For real-time encoding and streaming, FFMPEG software is widely used[8].The steps of preprocessing, source encoding and the broadcasting software reside on a laptop in the ambulance. Parameters involved in video streaming that includes packet loss rate, delay, jitter, bitrate, background traffic, mobility, end-user devices that is present in network simulators like OPNET (or) NS-2 software.

\section{Results And Discussion}

The efficiency of the proposed encoding scheme provides a comparative evaluation of two major concepts mainly of 1)FMO with constant QP video slices 2)FMO with variable QP in noisy environments. This provides the bitrate gains for perceptual quality which are being computed by using the BD-PSNR algorithm(see Fig.2).

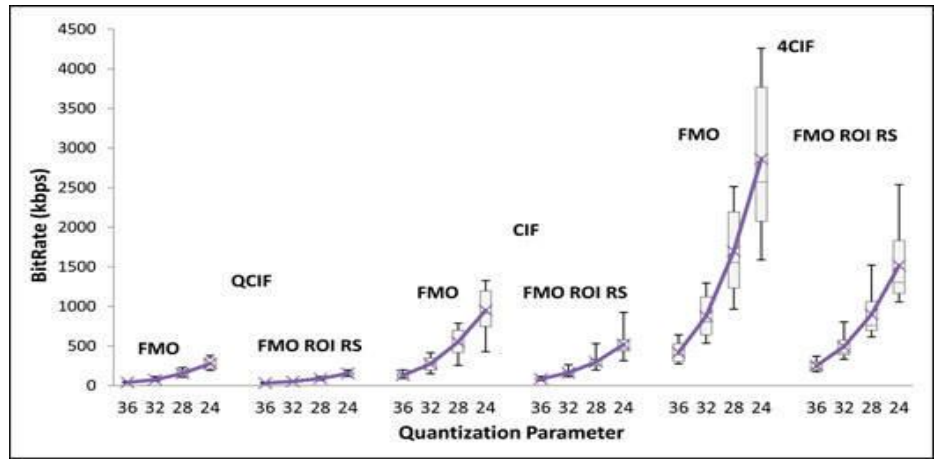

Fig.2.Boxplots depicting bitrate requirements for perceptual quality of different encoding schemes of QCIF,CIF and 4CIF

\section{A.Mobile WiMAX Medical video transmission}

The packet losses occur at large distances to the base station. But this condition is not suitable for QPSK1/2 scheme, as it exhibits a higher throughput performance irrespective of the channel's conditions[6]. The modulation scheme of QPSK1/2 requires lower signal-to-noise ratio (SNR) compared to 16-QAM3/4 and 64-QAM3/4 to maintain the quality(see Table I). As the mobile station (ambulance) moves away from the base station and hence the signal attenuation increases, the quality of the video is significantly reduced. In the clinical evaluation, the medical expert was asked to comment on the clinical video regarding the resolutions of both the type of CIF and 4CIF.The findings provided that the quality of $4 \mathrm{CIF}$ is better, as they assessed a detailed visualization of the plaque morphology, while in the lower CIF resolution, the type of plaque alone can be determined.

Relationship between clinical criteria and video resolution

TABLE II

\begin{tabular}{lccc}
\hline \hline & QCIF & CIF & 4CIF \\
\hline $\begin{array}{l}\text { Bit Rate } \\
\text { Gain (\%) }\end{array}$ & 34.7 & 39.8 & 42.3 \\
\hline \hline $\begin{array}{l}{ }^{1} \text { FMO ROI RS stands for variable quality slice encoding with redundant slices. FMO stands } \\
\text { for standard H.264/AVC FMO type 2 encoding. } \\
\text { Bitrate gains account for the reductions in bitrate requirements for equivalent objective } \\
\text { quality (PSNR), estimated using the BD-PSNR algorithm for QP values: } 36,32,28, \text { and } 24 .\end{array}$
\end{tabular}

\section{Concluding Remarks}

This paper derives the work done for transmission of the H.264/AVC video over mobile wireless medium. By reducing the bitrate, the quality of the video can be achieved, which was depicted in this paper. Even at speeds of $100 \mathrm{kmph}$ and at distance of $1 \mathrm{~km}$ from the base station, the transmission of the ultrasound video provides higher resolution and lower delay over mobile networks. Future work implies that the video transmission being simulated over Long-Term Evolution(LTE)and LTE-Advanced wireless channels using OPNET network simulator and NS-2.To improve the efficient error-resilient encoding, the emerging High Efficiency Video Coding(HEVC)standard is to be used to provide usage for other medical videos. 


\section{References}

[1] C.S. Pattichis, E. Kyriacou, S. Voskarides, M.S. pattichis, and R. Istepanian, "Wireless telemedicine systems:An overview,"IEEE Antennas Propag.Mag.,vol.44,no.2,pp. 143-153, Apr.2002.

[2] A. Panayides, M.S Pattichis, C.S. Pattichis, C.P. Loizou, M. Pantziaris, and A. Pitsillides, "Atherosclerotic plaque ultrasound video encoding, wireless transmission, and quality assessment using H.264."IEEE Trans.Inf.Technol.Biomed., vol.15, no.3,pp.387-397, May 2011.

[3] A. Panayides, M.S Pattichis, C.S Pattichis, A. Pitsillides, "A tutorial for emerging wireless medical video transmission systems," IEEE Antennas Propag.Mag., vol.53, no.2, pp.202-213, Apr.2011.

[4] A.S. Panayides, "Diagnostically resilient encoding, wireless transmission, and quality assessment of medical video,"Ph.D. Dissertation, Dept.Comput.Sci., Univ.Cyprus, Nicosia, Cyprus, 2011

[5] A. Panayides, M.S Pattichis, C.S Pattichis, C.N.Schizas, A. Spanias, and E.C. Kyriacou, "An overview of recent end-to-end wireless medical video telemedicine systems using 3G," in Proc. Annu. Int. Conf. IEEE Eng. Med. Biol. Soc., Buenos Aires, Argentina, Aug.31-sep.4, 2010, pp. 1045-1048.

[6] C.S Pattichis, C.P Loizou, and M. Pantziaris, And A. Nicolaides, "An integrated system for the segmentation of atherosclerotic carotid plaque,"IEEE Trans. Inf. Technol. Biomed., vol. 11,no. 5, pp. 661-667, Nov.2007.

[7] M.G. Martini and C.T.E.R. Hewage, "Flexible macroblock ordering for context-aware ultrasound video transmission over mobile WiMAX,” Int. J. Telemed. Appl., vol. 2010, p. 14, 2010.

[8] A.Alinejad, N. Philip, and R. Istepanian, "Cross layer ultrasound video streaming over mobile WiMAX and HSUPA networks," IEEE Trans. Inf. Technol. Biomed., vol. 16,no. 1, pp.31-39,Jan. 2012. 